\title{
The RBF Hyperparameter in Metamodel-Assisted Evolutionary Search
}

\author{
Yoel TENNE 1 \\ Mechanical Engineering Department, Ariel University, Israel
}

\begin{abstract}
RBF metamodels, which are commonly used in expensive optimization problems, rely on a hyperparameter which affects their prediction. The optimal hyperparameter value is typically unknown and hence needs to be estimated by additional procedures. As such this study examines if this overhead is justified from an overall search effectiveness perspective, namely, if changes in the hyperparameter yield significant performance differences. Analysis based on extensive numerical experiments shows that changes are significant in functions with low to moderate multimodality but are less significant in functions with highly multimodality.
\end{abstract}

Keywords. Evolutionary algorithms, Metamodels, RBF, Expensive functions

\section{Introduction}

Simulation-driven design optimization which is common in engineering and science defines presents unique challenges such as non-analytic 'black-box' functions, computationally expensive evaluations, and complicated function features [1]. A framework which has been extensively used for the solution of such problems is the metamodel (or surrogate) assisted evolutionary algorithm (EA) where the former is an interpolant trained based on sampled vectors (candidate designs). A common metamodel variant is the radial basis functions (RBF) predictor which is an aggregation of basis functions. The latter rely on a hyperparameter which defines their shape and therefore varying this parameter affects the metamodel prediction accuracy and possibly the overall search effectiveness. The optimal hyperparameter value is typically unknown and hence needs to be estimated. This raises the question if the required computational overhead is justified, namely, does it yield significant performance gains. Previous studies have examined the impact of hyperparameter calibration solely on the metamodel prediction accuracy $[2,3]$. However, the overall search effectiveness is affected in an intricate manner in which metamodel accuracy is just one component [4]. Accordingly this study takes a different approach and examines the impact of hyperparameter variation on the overall search effectiveness. The remainder of this paper is organized as follows: Section 2 surveys relevant studies, Section 3 describes the algorithm and numerical experiments, Section 4 presents the results and analysis, and lastly Section 5 concludes this paper.

\footnotetext{
${ }^{1}$ Corresponding Author: Mechanical Engineering Department, Ariel University, Israel, E-mail: y.tenne@ ariel.ac.il
} 


\section{Previous Studies}

Metamodel-assisted EAs have been used extensively in problems ranging from drug formulation to aircraft design [1]. Extensions ensembles of multiple metamodels [5] and [6] for incorporation of machine learning techniques.

The issue of the RBF hyperparameter calibration has been explored in multiple studies. Early examples include [7] which used a heuristic fixed value and [8] which used RMS error minimization. Related studies also used calibration based on error minimization but with a leave-one-out estimation approach [9,10,11]. Calibration based on a training-testing approach was explored by $[2,12]$. The studies mentioned have gauged the parameter calibration based solely on the metamodel prediction accuracy.

\section{Experiments Layout}

To study the impact of the hyperparameter numerical experiments were performed with an RBF metamodel-assisted EA with different hyperparameter settings. A representative EA was used which begins by sampling a random set of vectors and it then repeatedly: i) ranks the vectors by fitness, ii) selects parents by stochastic universal selection (SUS) and recombines them with a uniform crossover operator (probability of 0.7 ) to produce offspring, and iii) mutates the offspring (Breeder genetic algorithm operator, probability of 0.1) [13]. The population size was 20 and the generations limit was 1000 .

Also a standard RBF metamodel was used which is defined as

$$
m(\vec{x})=\sum_{i=1}^{n} \alpha_{i} \phi_{i}(\vec{x}), \phi_{i}(\vec{x})=\exp \left(-\kappa\left\|\vec{x}-\vec{x}_{i}\right\|_{2}\right)
$$

where $\phi_{i}(\vec{x})$ are Gaussian basis functions , $\kappa$ is the hyperparameter, and $\vec{x}_{i}, i=1 \ldots n$ are the sampled vectors. The coefficients $\alpha_{i}$ are obtained from the Lagrange interpolation conditions $m\left(\vec{x}_{i}\right)=f\left(\vec{x}_{i}\right)$, namely

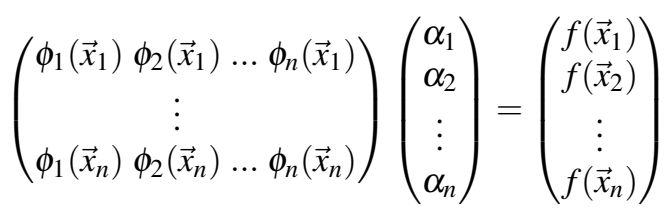

The complete RBF-EA algorithm operates as follows: it begins by sampling an initial random set of vectors and uses them to train an RBF metamodel. Next the main loop begins where the EA uses the metamodel predictions. Every $g$ generations the best $s$ vectors in the EA population are evaluated with the true objective function. The metamodel is then retrained and the EA population is re-evaluated with the new metamodel. The process stops when the limit of true (expensive) function evaluations or maximum EA generations has been reached. Following [14] the settings $g=4$ and $s=20 \%$ were used. To complete this description Algorithm 1 gives the full pseudocode. 
sample an initial random population and evaluate;

repeat

train an RBF metamodel based on sampled vectors;

run one generation of the EA;

foreach $g$ generation do

evaluate the best \%s vectors with the true function;

retrain the metamodel and re-evaluate the population;

end

until max true (expensive) evaluations or max generations;

Algorithm 1: The implemented RBF-EA algorithm.

\section{Experiments}

The above algorithm was applied to an established set of test functions (Ackley, Griewank, Powell, Rastrigin, and Rosenbrock) in dimensions 5 and 10 [15]. Four hyperparameter settings were tested: $0.01,0.1,1$, and 10, and for each functionhyperparameter combination 30 runs were repeated yielding a total of $5 \times 4 \times 30=600$ runs.

Tables 1 and 2 show the resultant statistics (mean, median, standard deviation, min, $\max$ ) of the best true function value found. The Mann-Whitney $p$-value is also included where the null hypothesis examined was $P(b>r)>0.5$ and $b$ are the results of the variant with the best mean and $r$ are the results of one of the other variants in turn. Statistical significance is accepted for $p<0.05$. From analysis of the results it follows that:

- Changing the hyperparameter yielded statistically significant performance differences for the Griewank, Powell, and Rosenbrock functions both in the 5D and 10D cases.

- Changes were not statistically significant for the Ackley and Rastrigin functions both in the 5D and 10D cases.

- The $\kappa=0.1$ setting was the best performing in 6 cases, followed by $\kappa=0.01$ and $\kappa=1$ settings in 2 cases each.

If follows that changes to the hyperparameter were had a significant impact with lowmedium multimodality functions. This is attributed to their simpler shape which allowed, given a suitable hyperparameter, for an adequate approximation. In contrast, high multimodality functions (Ackley, Rastrigin) hyperparameter changes had a markedly smaller impact on performance. This is attributed to their complex shape and consequentially poor metamodel accuracy which is less sensitive to different hyperparameter values. Figure 1 shows the median $p$-value by function (out of the three Mann-Whitney tests) and the division between the low and high multimodality functions is evident. Also, moderate hyperparameter values $(\kappa=0.1)$ typically yielded the best results since this avoided generating basis functions which are too narrow or too flat and hence cannot adequately approximate the test functions. Lastly, in the 10D cases the metamodel accuracy also degraded due to the higher dimension, namely it had a similar impact as multimodality.

To augment the analysis additional tests were performed with an application of airfoil shape optimization as a representative real-world simulation-driven problem. The goal is to find an airfoil which maximizes its lift to drag ratio 
Table 1. Statistics for the 5D functions

\begin{tabular}{|c|c|c|c|c|c|}
\hline Function & Statistic & $\kappa=0.01$ & $\kappa=0.1$ & $\kappa=1$ & $\kappa=10$ \\
\hline \multirow{6}{*}{ Ackley-05 } & Mean & $1.675 e+01$ & $1.813 \mathrm{e}+01$ & $1.815 \mathrm{e}+01$ & $1.728 \mathrm{e}+01$ \\
\hline & SD & $3.202 \mathrm{e}+00$ & $1.802 \mathrm{e}+00$ & $1.544 \mathrm{e}+00$ & $2.135 \mathrm{e}+00$ \\
\hline & Median & $1.768 \mathrm{e}+01$ & $1.838 \mathrm{e}+01$ & $1.845 \mathrm{e}+01$ & $1.785 \mathrm{e}+01$ \\
\hline & Min & $7.716 \mathrm{e}+00$ & $1.300 \mathrm{e}+01$ & $1.304 \mathrm{e}+01$ & $1.109 \mathrm{e}+01$ \\
\hline & $\operatorname{Max}$ & $2.066 \mathrm{e}+01$ & $2.027 \mathrm{e}+01$ & $2.005 \mathrm{e}+01$ & $2.015 \mathrm{e}+01$ \\
\hline & $p$ value & & $9.918 \mathrm{e}-02$ & $8.689 \mathrm{e}-02$ & $4.238 \mathrm{e}-01$ \\
\hline \multirow{6}{*}{ Griewank-05 } & Mean & $3.916 e+01$ & $3.084 \mathrm{e}+01$ & $2.480 \mathrm{e}+01$ & $3.267 \mathrm{e}+01$ \\
\hline & SD & $1.884 \mathrm{e}+01$ & $1.522 \mathrm{e}+01$ & $1.353 \mathrm{e}+01$ & $1.486 \mathrm{e}+01$ \\
\hline & Median & $3.961 \mathrm{e}+01$ & $2.750 \mathrm{e}+01$ & $2.167 \mathrm{e}+01$ & $2.907 \mathrm{e}+01$ \\
\hline & Min & $7.940 \mathrm{e}+00$ & $8.968 \mathrm{e}+00$ & $5.288 \mathrm{e}+00$ & $9.673 e+00$ \\
\hline & Max & $8.665 \mathrm{e}+01$ & $7.769 e+01$ & $6.050 \mathrm{e}+01$ & $7.108 \mathrm{e}+01$ \\
\hline & $p$ value & $1.281 \mathrm{e}-03$ & $3.926 \mathrm{e}-02$ & & $5.497 \mathrm{e}-03$ \\
\hline \multirow{6}{*}{ Powell-05 } & Mean & $2.885 \mathrm{e}+01$ & $1.540 \mathrm{e}+01$ & $1.364 \mathrm{e}+02$ & $1.227 \mathrm{e}+02$ \\
\hline & $\mathrm{SD}$ & $2.523 \mathrm{e}+01$ & $1.357 \mathrm{e}+01$ & $1.225 \mathrm{e}+02$ & $1.211 \mathrm{e}+02$ \\
\hline & Median & $2.116 \mathrm{e}+01$ & $8.740 \mathrm{e}+00$ & $9.514 \mathrm{e}+01$ & $7.374 \mathrm{e}+01$ \\
\hline & Min & $1.129 \mathrm{e}+00$ & $4.463 e-01$ & $1.281 \mathrm{e}+00$ & $2.206 \mathrm{e}+01$ \\
\hline & Max & $1.073 \mathrm{e}+02$ & $4.944 \mathrm{e}+01$ & $5.200 \mathrm{e}+02$ & $5.196 \mathrm{e}+02$ \\
\hline & $p$ value & $1.279 \mathrm{e}-02$ & & $1.607 \mathrm{e}-08$ & $2.658 \mathrm{e}-10$ \\
\hline \multirow{6}{*}{ Rastrigin-05 } & Mean & $4.263 \mathrm{e}+01$ & $3.902 \mathrm{e}+01$ & $4.299 e+01$ & $4.212 \mathrm{e}+01$ \\
\hline & SD & $1.136 \mathrm{e}+01$ & $1.103 \mathrm{e}+01$ & $1.311 \mathrm{e}+01$ & $9.976 \mathrm{e}+00$ \\
\hline & Median & $4.243 e+01$ & $4.025 \mathrm{e}+01$ & $4.409 e+01$ & $4.073 \mathrm{e}+01$ \\
\hline & Min & $1.966 \mathrm{e}+01$ & $1.706 \mathrm{e}+01$ & $7.767 \mathrm{e}+00$ & $2.789 \mathrm{e}+01$ \\
\hline & Max & $6.739 \mathrm{e}+01$ & $5.802 \mathrm{e}+01$ & $6.808 \mathrm{e}+01$ & $6.398 \mathrm{e}+01$ \\
\hline & $p$ value & $1.306 \mathrm{e}-01$ & & $1.044 \mathrm{e}-01$ & $1.797 \mathrm{e}-01$ \\
\hline \multirow{6}{*}{ Rosenbrock-05 } & Mean & $3.900 \mathrm{e}+01$ & $9.920 \mathrm{e}+00$ & $9.758 \mathrm{e}+01$ & $1.902 \mathrm{e}+02$ \\
\hline & $\mathrm{SD}$ & $5.200 \mathrm{e}+01$ & $1.293 \mathrm{e}+01$ & $9.729 \mathrm{e}+01$ & $7.089 \mathrm{e}+01$ \\
\hline & Median & $1.857 \mathrm{e}+01$ & $4.961 \mathrm{e}+00$ & $8.194 \mathrm{e}+01$ & $1.964 \mathrm{e}+02$ \\
\hline & Min & $3.040 \mathrm{e}+00$ & $3.772 \mathrm{e}-01$ & $3.574 \mathrm{e}+00$ & $7.153 e+01$ \\
\hline & Max & $2.396 \mathrm{e}+02$ & $5.355 e+01$ & $3.977 e+02$ & $3.336 \mathrm{e}+02$ \\
\hline & $p$ value & $2.108 \mathrm{e}-05$ & & $7.188 \mathrm{e}-07$ & $1.436 \mathrm{e}-11$ \\
\hline
\end{tabular}

The best mean is emphasized.

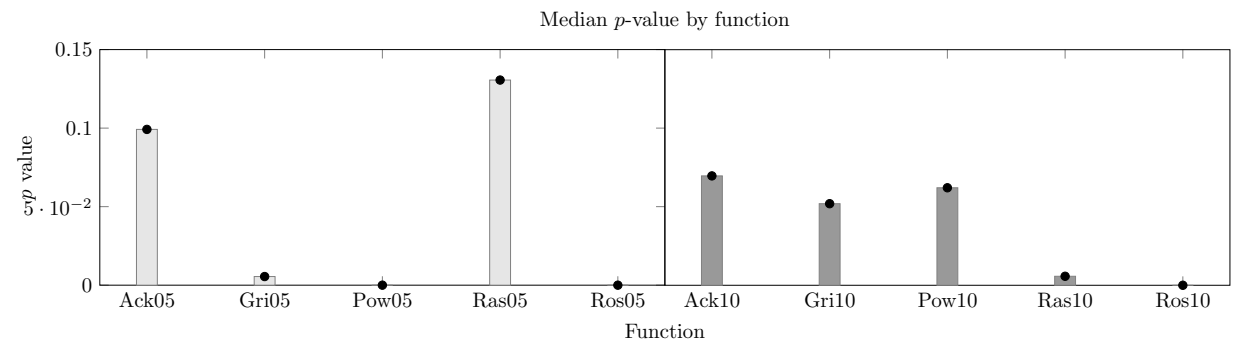

Figure 1. The median $p$-values of comparisons for each function.

$$
f=-\frac{c_{L}}{c_{D}}
$$

where the lift $\left(c_{L}\right)$ and drag $\left(c_{D}\right)$ coefficients were obtained from the XFoil numerical aerodynamic simulation code [16]. Airfoils were represented with the NACA 4 digit parameterization and standard flight conditions were used (Mach number=0.7, Reynolds number $=10^{7}$, angle of attack $=2^{\circ}$ ) and Figure 2 shows the problem layout and all other algorithm parameters were as before.

Table 3 shows the resultant statistics from which it follows that changes to the hyperparameter were not statistically significant (as with some of the test functions in the pre- 
Table 2. Statistics for the 10D functions

\begin{tabular}{|c|c|c|c|c|c|}
\hline Function & Statistic & $\kappa=0.01$ & $\kappa=0.1$ & $\kappa=1$ & $\kappa=10$ \\
\hline \multirow{6}{*}{ Ackley-10 } & Mean & $1.973 \mathrm{e}+01$ & $2.011 \mathrm{e}+01$ & $1.972 \mathrm{e}+01$ & $1.983 \mathrm{e}+01$ \\
\hline & SD & $1.217 \mathrm{e}+00$ & $4.317 \mathrm{e}-01$ & $5.775 \mathrm{e}-01$ & $4.632 \mathrm{e}-01$ \\
\hline & Median & $2.004 \mathrm{e}+01$ & $2.016 \mathrm{e}+01$ & $1.965 \mathrm{e}+01$ & $1.985 \mathrm{e}+01$ \\
\hline & Min & $1.498 \mathrm{e}+01$ & $1.923 \mathrm{e}+01$ & $1.800 \mathrm{e}+01$ & $1.824 \mathrm{e}+01$ \\
\hline & $\operatorname{Max}$ & $2.095 \mathrm{e}+01$ & $2.083 \mathrm{e}+01$ & $2.055 \mathrm{e}+01$ & $2.059 \mathrm{e}+01$ \\
\hline & $p$ value & $6.964 \mathrm{e}-02$ & $3.118 \mathrm{e}-03$ & & $2.577 \mathrm{e}-01$ \\
\hline \multirow{6}{*}{ Griewank-10 } & Mean & $1.482 \mathrm{e}+02$ & $1.129 \mathrm{e}+02$ & $1.156 \mathrm{e}+02$ & $1.244 \mathrm{e}+02$ \\
\hline & SD & $3.170 \mathrm{e}+01$ & $3.293 \mathrm{e}+01$ & $3.056 \mathrm{e}+01$ & $3.174 \mathrm{e}+01$ \\
\hline & Median & $1.529 \mathrm{e}+02$ & $1.046 \mathrm{e}+02$ & $1.093 \mathrm{e}+02$ & $1.313 e+02$ \\
\hline & Min & $8.037 \mathrm{e}+01$ & $5.000 \mathrm{e}+01$ & $6.540 \mathrm{e}+01$ & $5.481 \mathrm{e}+01$ \\
\hline & Max & $1.979 \mathrm{e}+02$ & $1.939 \mathrm{e}+02$ & $2.057 \mathrm{e}+02$ & $1.710 \mathrm{e}+02$ \\
\hline & $p$ value & $6.429 \mathrm{e}-05$ & & $3.894 \mathrm{e}-01$ & $5.194 e-02$ \\
\hline \multirow{6}{*}{ Powell-10 } & Mean & $6.392 \mathrm{e}+01$ & $6.374 \mathrm{e}+01$ & $1.482 \mathrm{e}+02$ & $8.858 \mathrm{e}+01$ \\
\hline & SD & $5.593 \mathrm{e}+01$ & $6.411 \mathrm{e}+01$ & $9.066 \mathrm{e}+01$ & $7.040 \mathrm{e}+01$ \\
\hline & Median & $5.303 e+01$ & $4.660 \mathrm{e}+01$ & $1.514 \mathrm{e}+02$ & $6.497 \mathrm{e}+01$ \\
\hline & Min & $8.446 \mathrm{e}+00$ & $7.498 \mathrm{e}+00$ & $1.346 \mathrm{e}+01$ & $5.057 \mathrm{e}+00$ \\
\hline & Max & $2.532 \mathrm{e}+02$ & $3.408 \mathrm{e}+02$ & $3.492 \mathrm{e}+02$ & $2.581 \mathrm{e}+02$ \\
\hline & $p$ value & $4.941 \mathrm{e}-01$ & & $2.717 \mathrm{e}-05$ & $6.208 \mathrm{e}-02$ \\
\hline \multirow{6}{*}{ Rastrigin-10 } & Mean & $1.057 \mathrm{e}+02$ & $1.190 \mathrm{e}+02$ & $1.232 \mathrm{e}+02$ & $1.093 \mathrm{e}+02$ \\
\hline & $\mathrm{SD}$ & $1.925 \mathrm{e}+01$ & $1.618 \mathrm{e}+01$ & $1.582 \mathrm{e}+01$ & $1.734 \mathrm{e}+01$ \\
\hline & Median & $1.083 \mathrm{e}+02$ & $1.184 \mathrm{e}+02$ & $1.240 \mathrm{e}+02$ & $1.091 \mathrm{e}+02$ \\
\hline & Min & $6.402 \mathrm{e}+01$ & $8.002 \mathrm{e}+01$ & $7.702 \mathrm{e}+01$ & $6.161 \mathrm{e}+01$ \\
\hline & Max & $1.310 \mathrm{e}+02$ & $1.564 \mathrm{e}+02$ & $1.532 \mathrm{e}+02$ & $1.441 \mathrm{e}+02$ \\
\hline & $p$ value & & $5.733 e-03$ & $1.461 \mathrm{e}-04$ & $4.008 \mathrm{e}-01$ \\
\hline \multirow{6}{*}{ Rosenbrock-10 } & Mean & $1.427 \mathrm{e}+02$ & $1.162 \mathrm{e}+02$ & $1.231 \mathrm{e}+03$ & $1.107 \mathrm{e}+03$ \\
\hline & SD & $1.132 \mathrm{e}+02$ & $6.780 \mathrm{e}+01$ & $4.217 \mathrm{e}+02$ & $3.513 e+02$ \\
\hline & Median & $1.263 \mathrm{e}+02$ & $1.037 \mathrm{e}+02$ & $1.227 \mathrm{e}+03$ & $1.019 \mathrm{e}+03$ \\
\hline & Min & $3.131 \mathrm{e}+01$ & $2.922 \mathrm{e}+01$ & $2.908 \mathrm{e}+02$ & $4.837 \mathrm{e}+02$ \\
\hline & Max & $6.257 \mathrm{e}+02$ & $3.315 \mathrm{e}+02$ & $2.141 \mathrm{e}+03$ & $1.954 \mathrm{e}+03$ \\
\hline & $p$ value & $1.997 \mathrm{e}-01$ & & $1.588 \mathrm{e}-11$ & $1.436 \mathrm{e}-11$ \\
\hline
\end{tabular}

The best mean is emphasized.

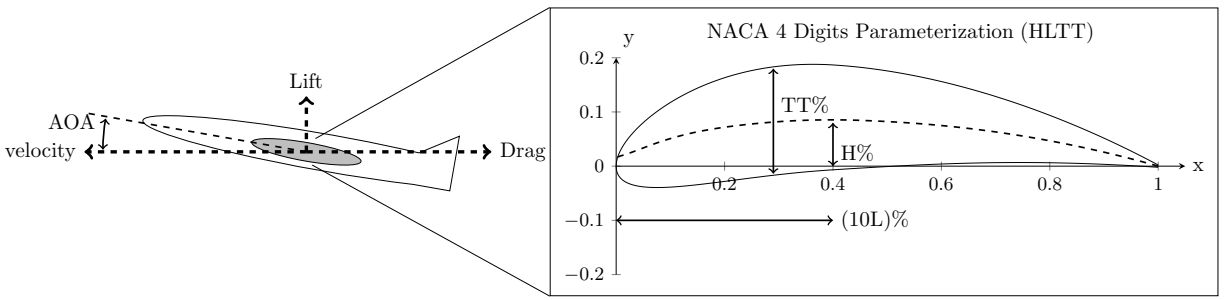

Figure 2. The airfoil problem with the NACA 4 digit parameterizaiton.

vious section) which indicates that the objective function has a complicated landscape. This was confirmed during the tests by the fact that the simulation failed to evaluate numerous candidate airfoils, namely, the function is discontinuous and possibly highly multimodal. Results also show that the best performance was observed with $\kappa=0.01$ which is consistent with the earlier results.

\section{Conclusion}

The RBF metamodel-assisted EA has been widely used in the literature with simulationdriven optimization problems. This metamodel relies on a hyperparameter which affects 
Table 3. Statistics for the airfoil problem

\begin{tabular}{lrrrr} 
Statistic & \multicolumn{1}{c}{$\kappa=0.01$} & \multicolumn{1}{c}{$\kappa=0.1$} & \multicolumn{1}{c}{$\kappa=1$} & \multicolumn{1}{c}{$\kappa=10$} \\
\hline Mean & $-\mathbf{1 . 8 9 6 e}+\mathbf{0 2}$ & $-1.811 \mathrm{e}+02$ & $-1.728 \mathrm{e}+02$ & $-1.682 \mathrm{e}+02$ \\
SD & $5.471 \mathrm{e}+01$ & $5.700 \mathrm{e}+01$ & $6.368 \mathrm{e}+01$ & $5.712 \mathrm{e}+01$ \\
Median & $-2.084 \mathrm{e}+02$ & $-1.931 \mathrm{e}+02$ & $-1.939 \mathrm{e}+02$ & $-1.601 \mathrm{e}+02$ \\
Min & $-2.586 \mathrm{e}+02$ & $-2.564 \mathrm{e}+02$ & $-2.662 \mathrm{e}+02$ & $-2.564 \mathrm{e}+02$ \\
Max & $-7.424 \mathrm{e}+01$ & $-5.714 \mathrm{e}+01$ & $-1.436 \mathrm{e}+01$ & $-8.499 \mathrm{e}+01$ \\
$p$ value & & $1.976 \mathrm{e}-01$ & $1.855 \mathrm{e}-01$ & $8.457 \mathrm{e}-02$ \\
\hline
\end{tabular}

its prediction. This study examined if changes to the hyperparameter affected the overall search effectiveness and therefore if calibration is justified. Results from an extensive set of numerical experiments show that hyperparameter changes had a significant impact with low-medium multimodality functions and lesser impact in high multimodality functions. Moderate hyperparameter values (around 1) typically performed best.

\section{References}

[1] Tenne Y, Goh CK, editors. Computational Intelligence in Expensive Optimization Problems. vol. 2 of Evolutionary Learning and Optimization. Berlin: Springer; 2010.

[2] Durantin C, Rouxel J, Désidéri JA, Gliére A. Multifidelity surrogate modeling based on radial basis functions. Structural and Multidisciplinary Optimization. 2017;56:1061-1075.

[3] Bengisen PG. A New Regression-Based Approach to Estimate the Shape Parameter of MQ-RBFs in a Free Convection Problem. Journal of Computing and Information Science in Engineering. 2019;20(1). 011009.

[4] Ong YS, Zhou Z, Lim D. Curse and Blessing of Uncertainty in Evolutionary Algorithm Using Approximation. In: Proceedings of the IEEE Congress on Evolutionary Computation (CEC). Piscataway, New Jersey: IEEE; 2006. p. 2928-2935.

[5] Tenne Y. An Adaptive-topology Ensemble Algorithm for Engineering Optimization Problems. Optimization and Engineering. 2015;16(2):303-334.

[6] Tenne Y. Frameworks for simulation-driven optimization. Journal of Applied Mathematics and Computer Science. 2017;27(1):105-118.

[7] Hardy RL. Theory and Applications of the Multiquadric-Biharmonic Method. Computers and Mathematics with Applications. 1990;19(8/9):163-208.

[8] Kansa EJ, Carlson RE. Improved Accuracy of Multiquadric Interpolation Using Variable Shape Parameters. Computers and Mathematics with Applications. 1992;24(12):99-120.

[9] Rippa S. An Algorithm for Selecting a Good Value for the Parameter $c$ in Radial Basis Function Interpolation. Advances in Computational Mathematics. 1999;11(2-3):193-210.

[10] Hickernell FJ, Hon YC. Radial Basis Functions Approximation of the Surface Wind Field from Scattered Data. International Journal of Applied Science and Computation. 1998;4(3):221-247.

[11] Sóbester A, Leary SJ, Keane AJ. On the Design of Optimization Strategies Based on Global Response Surface Approximation Models. Journal of Global Optimization. 2005;33:31-59.

[12] Marchetti F. The extension of Rippa's algorithm beyond LOOCV. Applied Mathematics Letters. 2021;120:107262.

[13] Chipperfield A, Fleming P, Pohlheim H, Fonseca C. Genetic Algorithm TOOLBOX For Use with MATLAB, Version 1.2. Sheffield; 1994.

[14] Ratle A. Kriging as a surrogate fitness landscape in evolutionary optimization. Artificial Intelligence for Engineering Design, Analysis and Manufacturing. 2001;15(1):37-49.

[15] Suganthan PN, Hansen N, Liang JJ, Deb K, Chen YP, Auger A, et al. Problem Definitions and Evaluation Criteria for the CEC 2005 Special Session on Real-Parameter Optimization; 2005. KanGAL 2005005.

[16] Drela M, Youngren H. XFOIL 6.9 User Primer. Cambridge, MA: Massachusetts Institute of Technology; 2001. 